\title{
La dimensión cognitiva de las emociones en la vida moral: los aportes de Martha Nussbaum al estado actual de la discusión*
}

\author{
The cognitive dimension of emotions in the moral life: the \\ contributions of Martha Nussbaum to the current state of the \\ discussion
}

\author{
Iván Alfonso Pinedo-Cantillo ** \\ Universidad Nacional de Colombia \\ Jaime Yáñez-Canal *** \\ Universidad Nacional de Colombia
}

Recepción: 10 de marzo del 2017

Evaluación: 27 de marzo del 2017

Aceptación: 4 de mayo del 2017

\footnotetext{
* Grupo de investigación estudios sobre el desarrollo socio-moral. Departamento de Psicología. Universidad Nacional de Colombia.

** Filósofo, Magíster en Filosofía, Magíster en Educación, Candidato a Doctor en Filosofía. Miembro del Grupo de Investigación "Estudios sobre el desarrollo socio-moral", Universidad Nacional de Colombia. iapinedoc@unal.edu.co

*** Psicólogo, Doctor en filosofía. Profesor Asociado Dpto. de Psicología, Universidad Nacional de Colombia. Grupo de Investigación "Estudios sobre el desarrollo socio moral”. jyanezc@unal.edu.co
} 
La dimensión cognitiva de las emociones en la vida moral: los aportes de Martha Nussbaum al estado de la discusión. Iván A. Pinedo-Cantillo y Jaime Yañes-Canal. Pág.: 105 - 127

\title{
Resumen
}

La relación entre emociones y vida moral constituye hoy en día un amplio campo de investigación en filosofía y psicología. Al respecto, Martha Nussbaum ofrece importantes puntos de reflexión que nos llevan a preguntarnos por aquellas facultades cognitivas que estructuran las emociones morales, como la compasión, y diferencian radicalmente la expresión emocional humana de la de animales no humanos. La inclusión de creencias y actos valorativos como elementos constitutivos de las emociones morales hacen que la propuesta de Nussbaum se oriente hacia una disolución de la vieja dicotomía moderna entre razón y emoción, a la vez que posibilita una mejor comprensión del valor ético de las emociones como informadoras de vulnerabilidad y daño moral. De esta manera, la perspectiva cognitivo-evaluadora de Nussbaum ingresa a la actual discusión sobre cómo las emociones pueden influir en nuestros juicios y decisiones morales en medio de un mundo conflictivo, inequitativo y que genera sufrimiento, pero que, no obstante, tiene aún posibilidades de consolidar sociedades justas, democráticas y plurales.

Palabras clave: Cognición; compasión; emoción; Nussbaum.

\begin{abstract}
Abstrac
The relationship between emotions and moral life is a wide subject of investigation nowadays among philosophical and psychological fields. Regarding such relationship, Martha Nussbaum contributes important points for reflecting that lead us to question ourselves about those cognitive faculties that structure our moral emotions -such as compassion- and radically make human emotional expression different from non-human animals. Beliefs and value judgments as constituting elements of moral emotions make Nassbaum's proposal address to a dissolution of the old still modern dichotomy between reason and emotion while it facilitates the understanding of emotional ethical values as announcers of vulnerability and moral damage. In that manner, Nussbaum's cognitive-assessor perspective turns out to be part of the current discussion of how emotions can influence our judgment and moral decisions in the middle of a violent, inequitable world that generates suffering but still, that could consolidate fair, democratic, and plural societies.
\end{abstract}

Key words: Cognition, Compassion, Emotion, Moral emotions, Nussbaum.

\section{Introducción}

En la filosofía y la psicología actual existe una discusión de vital importancia para la comprensión del ser humano; se trata de la relación entre las emociones y la moral y del vínculo de estas dimensiones con la razón. Aunque es un problema que podemos rastrear a lo largo de la historia de la filosofía, como reflexión que se reedita en diferentes épocas con distintos matices y orientaciones, en las últimas décadas se evidencia un renovado interés e 
intensos debates en torno a diversos planteamientos que intentan desentrañar la naturaleza y los rasgos definitorios de las emociones y la relevancia que estas pueden tener en distintos ámbitos de la vida humana, como son la deliberación moral, la educación y la vida pública de los ciudadanos. Estos nuevos enfoques actualizan, resignifican y cuestionan las posturas filosóficas tradicionales relacionadas con la comprensión de las emociones, y nos abren a horizontes inéditos de investigación que son de gran valor para el avance del conocimiento.

La historia de la relación entre las emociones y la moral puede remontarse hasta la época antigua, al igual que muchas de las posturas que de manera cercana a este problema tratan de establecer vínculos entre estos dos conceptos con la razón. En la filosofía clásica griega y romana encontramos tanto posturas que condenan las emociones, por librar una batalla con la razón en la lucha por el control de la psique humana, como aquellas que rescatan su participación en la determinación de la conducta moral y las proponen como fuente de armonía y de dimensiones creativas en el actuar humano. Platón, por ejemplo, es recordado por concebir las pasiones como caballos descarriados que debían ser controlados por el auriga, que personificaba el intelecto. La relación entre razón y emoción aparece, entonces, desde la antigüedad, como una cuestión central, tanto para el estudio de las emociones como para la comprensión de la justificación moral, los juicios y los valores.

A pesar de existir desde tiempos antiguos posturas que abogaban por integrar las emociones y la razón, y por incorporar estas expresiones en el comportamiento moral, las posturas que han dominado en nuestra historia occidental han sido las que defienden las dicotomías y oposiciones entre estos dos procesos. Estas posturas han estado teñidas por una óptica intelectualista dualista y excluyente que considera las dimensiones de la experiencia cognitiva, intelectual o racional como separadas y superiores a las de la emocional o sentimental (Cabezas, 2014); sin embargo, esta perspectiva dicotómica tiende en la actualidad a su disolución con el surgimiento de nuevos planteamientos que establecen una interdependencia entre cognición y emociones, y una relación estrecha entre emociones y vida moral. Estas propuestas de integración proponen el término cognición, como un concepto más abarcador que el de razón, pues incluye, además de este último, todo proceso de selección y organización de la información que es necesario para responder a los estímulos del ambiente.

En este contexto de "cognitivismo emocional", los planteamientos de Martha Nussbaum resultan relevantes para comprender la experiencia emocional como un tipo de conocimiento análogo al conocimiento racional; de ahí su insistencia en lo que denomina una "inteligencia de las emociones". Esta perspectiva filosófica establece nuevas miradas de lo cognitivo, en las cuales los actos de valoración, las creencias y la consideración de aspectos referidos a la dignidad humana y a su fragilidad son tenidos en cuenta como elementos que configuran la relación entre las emociones y el conocimiento moral. Particularmente, el análisis que esta filósofa realiza en torno a la naturaleza de la compasión representa un 
importante aporte a los debates actuales en torno al conjunto de procesos cognitivos que determinan y diferencian la expresión emocional de los seres humanos de aquella que se da en otras especies.

La postura cognitivo-evaluadora de Nussbaum nos enfrenta, entonces, a diferentes concepciones sobre la naturaleza de las emociones, a la conexión entre las emociones y los juicios morales y a la propiedad cognitiva de las emociones morales como informadoras de vulnerabilidad y sufrimiento inmerecido. En este artículo se busca, por tanto, exponer algunos puntos de reflexión que nos permiten comprender los aportes de Martha Nussbaum a la actual discusión sobre la relación entre moral y emociones, y las perspectivas de análisis que se abren frente a la acción moral y la formación de ciudadanos que aspiran a la justicia en medio de sociedades democráticas, abiertas y plurales.

Para alcanzar nuestro objetivo conviene reconocer brevemente cómo se llega a los actuales debates sobre la relación entre las emociones y la vida moral.

\section{De la dicotomía moderna entre razón y emoción a las teorías contemporáneas de la emoción}

En la tradición de la filosofía moral se asume la dicotomía entre razón y emoción según dos posturas centrales forjadas en el seno de la modernidad: la primera, representada por Hume y los sentimentalistas británicos, y la segunda, por Kant. En el contexto del siglo XVIII, tanto Hume como Kant buscaban una respuesta al problema del fundamento de la moral: cómo podíamos tener un conocimiento de lo moral y cómo podíamos dar cuenta de la obligación y la motivación para actuar sin tener que recurrir a una autoridad eclesiástica que definiera los conceptos y principios que debían orientar nuestro comportamiento. Para responder a este problema, ambos autores defenderán, desde diferentes perspectivas y con diversos argumentos, que el orden moral surge, de alguna manera, de la naturaleza humana (bien de la razón, bien del sentimiento) y de las exigencias de nuestra vida conjunta en sociedad. Este es el núcleo en donde se origina la discusión y dicotomía en filosofía moral entre razón y emoción que atravesará buena parte del pensamiento contemporáneo.

El análisis moral realizado por Hume intenta demostrar cómo la razón por sí sola no puede ser un motivo que influya en nuestra conducta; por el contrario, fundamenta lo moral en aspectos emocionales y asigna a la razón un papel secundario que se limita a establecer relaciones entre eventos o a identificar los mejores medios para alcanzar determinados fines, pero opera de manera fría, sin actos de valoración. En la Disertación sobre las pasiones nos dice este filósofo: "La razón, en un sentido estricto, significando el discernimiento de la verdad y la falsedad, no puede nunca por sí misma ser un motivo para la voluntad, y no puede tener influencia alguna sino en cuanto afecte a alguna pasión o afección” (Hume, 2004, p. 
161). Lo anterior quiere decir que la razón es incapaz de motivar nuestra conducta; si el sentimiento no desempeñara un papel importante en el conocimiento moral, nunca estaríamos motivados a hacer lo correcto y a evitar lo incorrecto. Este análisis llevó a Hume a plantear su polémica tesis según la cual la razón es, y debe ser, esclava de las pasiones. La emoción, para el filósofo escocés, es la que realiza el acto de valoración que conduce a condenar o a aprobar determinada conducta desde un punto de vista moral (Seoane, 2004). En el Tratado de la naturaleza humana (3.1.1), Hume es explícito en afirmar que no es la razón, sino el sentimiento, lo que constituye la base de las distinciones morales:

Puesto que el vicio y la virtud no pueden descubrirse solamente por la razón o la comparación de ideas, debe ser mediante alguna impresión o sentimiento que nos ocasionan por lo que somos capaces de fijar la diferencia entre ellos (...). La moralidad, por consiguiente, es más propiamente sentida que juzgada, aunque este sentimiento o afección es comúnmente tan suave y sutil que nos inclinamos a confundirlo con una idea (Hume, 1985, p. 297).

En contraste con esta postura, Kant defiende el papel de la razón como la base de todo juicio y acción moral. En este sentido, lo moral, al preocuparse por el deber, tiene que orientarse según criterios de razones explícitas: el ser humano debe plantearse de manera consciente aquellas normas que valdrían para todo tiempo y lugar, independientemente de sus intereses, sus simpatías o los vínculos afectivos con la situación o personas involucradas. El deber, en este sentido, demanda una formulación de principios universales y una decisión racional que orientaría la acción por encima de los intereses de las personas afectadas o de las consecuencias que pueda causar una determinada acción moral. Las emociones, en el contexto de los planteamientos de Kant, serían intereses o deseos subjetivos que no sirven para justificar moralmente una conducta: por ejemplo, no es ni puede ser por el sentimiento de compasión por el que nuestra conducta merezca una buena calificación moral, sino por el sabernos respetuosos de una máxima que ha sido convertida en legítima tras sobrepasar el examen de la universalidad.

La posición de Kant al respecto varía en matices de una obra a otra sin abandonar nunca sus argumentos en contra de una moral centrada en las emociones. En la Fundamentación de la metafísica de las costumbres podemos leer:

En cambio, el sentimiento moral, ese supuesto sentido especial, aunque es harto superficial la apelación a este sentido, con la creencia de que quienes no puedan pensar habrán de dirigirse bien por medio del sentir, en aquello que se refiere a meras leyes universales, y aunque los sentimientos, que por naturaleza son infinitamente distintos unos de otros en el grado, no dan una pauta igual del bien y del mal, y no 
puede uno por su propio sentimiento juzgar válidamente a los demás (Kant, 2013, p. 59).

Para Kant, actuar de manera ética significa actuar desinteresadamente, sin emociones o afectos, sino por respeto, convencimiento y amor al deber; podemos referiremos a un ejemplo para ilustrar la argumentación y la concepción de razón que ofrece el filósofo. Kant, en su preocupación de establecer los principios morales y el componente de la universalización como criterio esencial de la moral, presenta el caso de una persona que le pide a alguien prestado un dinero, con la promesa de que va a pagar su deuda, sin que realmente sea sincero con el cumplimiento de su compromiso; si este sujeto tiene como máxima mentir para conseguir sus objetivos puede condenarse en términos morales, ya que una norma personal de esa naturaleza no puede convertirse en universal; si esa máxima se convierte en universal, es claro que nadie podría creer en la sinceridad de las palabras que alguien dice en el momento de prometer pagar sus deudas; por lo tanto, el principio de universalización, característico del imperativo categórico de Kant, condena la mentira y el que alguien no pague sus deudas a pesar de prometerlo. Con el anterior ejemplo se ilustra la idea de razón que tenía Kant en su visión de la moral: la razón se establece con juicios explícitos y con cálculos sobre las consecuencias o implicaciones que pueden traer ciertas normas.

Independientemente de los ejemplos que Hume y Kant esgrimen en sus obras para ilustrar algún aspecto de lo moral y de su preocupación para determinar la idea de deber o el aspecto motivacional en la acción moral, es claro que la oposición entre razón y emoción se debe a la caracterización dicotómica de estas dos categorías (Cabezas, 2014); es decir, a las maneras opuestas cómo se han definido los dos términos. A partir de estas visiones opuestas en torno a la relación entre razón y emoción podemos entender la historia posterior que se desarrolló en filosofía y psicología para esclarecer este problema.

En la filosofía contemporánea, sin embargo, las emociones reciben un tratamiento distinto como consecuencia de la aparición de nuevos enfoques teóricos y de la participación de otras ciencias interesadas en este objeto de estudio. Se atribuye a Darwin un importante giro en la reflexión sobre las emociones al incorporar en su análisis diversos principios científicos evolucionistas que aparecen plasmados en su influyente obra La expresión de las emociones en los animales y en el hombre, publicada en 1872, y también se reconocen los aportes del médico y filósofo Thomas Brown a la consolidación de una teoría de las emociones con su texto Lecturas sobre la filosofía de la mente humana, reflexión que tuvo gran influencia entre los académicos a mediados del siglo XIX (Dixon, 2010). Estas obras hicieron que el problema moral de las pasiones y su relación con la razón se transformara en un problema filosófico y psicológico independiente que exigía clarificar preguntas relevantes en torno a la naturaleza de las emociones, tales como: ¿Qué es una emoción?, ¿qué cuenta como una emoción?, ¿cuáles emociones son básicas?, ¿cuáles son los rasgos definitorios de 
las emociones? y ¿cuáles son los componentes de la experiencia emocional? Surge, entonces, la necesidad de una descripción más amplia de la emoción o de teorías de la emoción que ayuden a distinguir entre emociones y otros fenómenos mentales, así como también a su clasificación dentro de tipos genéricos (Plamper, 2015). La emoción, por consiguiente, se convierte en un campo de estudio específico que interesa tanto a filósofos como a psicólogos, antropólogos y, más recientemente, a los investigadores provenientes de las denominadas neurociencias. En este contexto, el término pasión, característico de la reflexión filosófica antigua, medieval y moderna, es sustituido paulatinamente por el concepto secular de emoción, ampliamente utilizado por los nuevos enfoques teóricos (Dixon, 2003).

Las primeras reflexiones desarrolladas desde la psicología y la filosofía contemporánea de las emociones asumieron estos fenómenos como una serie de sensaciones somáticas y cambios fisiológicos involuntarios. Estas teorías enfatizan el elemento reactivo automático (pathos) de las emociones, entablando una conexión con la postura tradicional heredada de los griegos, en la cual las emociones son pasivas, esto es Pathe, pasiones, algo que nos sobreviene sin la intervención de la voluntad (Cabezas, 2014). En este sentido, las emociones son consideradas el resultado de una activación corporal específica que surge frente a situaciones desencadenantes particulares, por lo que para poder experimentar la emoción deben primero experimentarse los cambios corporales que han sido iniciados directamente por el estímulo percibido (Cornelius, 1996).

William James es uno de los pioneros de estas propuestas fisiológicas, no cognitivas, de la emoción; este autor afirma radicalmente que los "disturbios" corporales no son manifestación de un estado emocional previo, sino que dicho estado es posterior a la percepción de las reacciones físicas; en su célebre artículo ¿Qué es una emoción?, publicado en la revista Mind de 1884, defiende la reacción fisiológica como el elemento central para la emoción. El psicólogo Danés C.G. Lange estaba por esos años desarrollando una teoría similar, de ahí que esta propuesta se ha denominado, en forma conjunta, teoría de JamesLange, la cual ha ejercido notable influencia sobre la psicología y la filosofía de las emociones. Si bien las ideas de James han sido fuertemente criticadas, hay un consenso generalizado en que es un punto de referencia para todo aquel que quiera comprender el fenómeno emocional.

Según la teoría de James-Lange, las emociones son percepciones de los cambios fisiológicos y viscerales que tienen lugar en el individuo tras confrontarse con un estímulo. El modelo que sigue esta orientación teórica es el reflejo: reacciones automáticas que se desencadenan en presencia de un objeto externo que influye en nuestra percepción. Por ejemplo, cuando nos tropezamos de improviso con una serpiente, nuestros músculos se contraen involuntariamente y nuestra respiración se acelera, preparándonos instintivamente 
para huir, y el temor a la serpiente no es otra cosa que la percepción de estos cambios fisiológicos involuntarios e instintivos (Calhoun \& Solomon, 1989).

Particularmente, la hipótesis que proponen estos autores cambia el orden en el que hasta el momento se comprendían las emociones, en donde primero hay una percepción de algún hecho u objeto que excita el afecto mental llamado emoción y, luego, dicho estado mental produce una expresión corporal: sudoración, enrojecimiento, latidos rápidos. En esta nueva teoría los cambios corporales siguen directamente a la percepción del hecho excitante, y nuestro sentimiento de esos mismos cambios es la emoción (Plutchik, 1980).

La propuesta fisiológica de James-Lange se resume esquemáticamente en la secuencia: Percepción-Respuesta corporal-Emoción, en contraste con la idea tradicional cartesiana de Percepción-Emoción-Reacción corporal; se trata de una propuesta que partía del hecho de que las emociones suelen ir acompañadas de respuestas físicas, como el aceleramiento de los latidos del corazón, el temblor o las lágrimas, sensaciones que podemos percibir en el interior de nuestro cuerpo de forma similar a como captamos lo que pasa en el mundo exterior. Según lo anterior, James y Lange proponen que sentimos tristeza porque lloramos, y estamos asustados porque temblamos, y no al revés. La percepción de la reacción fisiológica es, por consiguiente, el elemento central de la emoción: "Es imposible pensar qué tipo de emoción de temor, por ejemplo, quedaría si no estuvieran presentes ni la aceleración de los latidos del corazón, ni la respiración entrecortada, ni el temblor de los labios o la laxitud de los miembros, ni la carne de gallina, ni las contracciones de las vísceras" (Calhoun \& Solomon, 1989, p. 147).

\section{Aproximaciones cognitivas al estudio de la emoción.}

En oposición a la teoría de James-Lange encontramos otro tipo de argumentos, no dicotómicos, como los propuestos por los psicólogos y filósofos Arnold (1960), Frijda (1987), Lazarus (1991), Ben Ze'ev (2000), Solomon (2003) y Nussbaum (2008), que intentan explicar las emociones como procesos cognitivos. Estos autores contemporáneos afirman que el cambio físico en sí mismo no es suficiente para explicar la diversidad y la riqueza de la expresión emocional humana; por ejemplo, si estamos en el bosque y sale súbitamente un oso, es claro que corremos y notamos las palpitaciones del corazón, pero ¿qué nos hace huir del peligro?, ¿qué pasa entre el estímulo y la respuesta?; tiene que haber un proceso que nos haga saber que el oso es una fuente de peligro y nos permita prever las respuestas adecuadas al peligro que puede ocurrir (Le Doux, 1999). Para estos autores es necesario, por tanto, que entre la activación fisiológica y la emoción se dé la mediación de un proceso de evaluación cognitiva, o appraisal, del cual depende que emerja o no una emoción.

Para Magda Arnold, la autora que acuñó el concepto de evaluación en los años sesenta del siglo pasado, hay un asunto que la teoría de James-Lange no explica satisfactoriamente: 
la idea según la cual tenemos la percepción de un objeto y luego cambios corporales que elicitan una emoción no permite establecer la conexión entre la percepción y la reacción corporal. Según Arnold (1960) falta una aclaración de lo que allí sucede, y es que la percepción del objeto está acompañada de una valoración (appraisal), que debe entenderse como un tipo de "juicio sentido", directo, inmediato, intuitivo (no equivalente a juicio intelectual o pensamiento racional, deliberado y consciente) de lo bueno o beneficioso que hay en una situación, o de lo malo o perjudicial, que debe rechazarse y que, en consecuencia, provoca la reacción emocional. Se trata de un proceso de evaluación que ocurre inconscientemente, pero cuyos efectos se graban en la conciencia como sentimiento emocional. En el ejemplo del oso del bosque percibimos el oso y lo evaluamos inconscientemente, y la experiencia consciente del miedo es resultado de la tendencia a huir. En este sentido, la secuencia para desencadenar las emociones, tal y como proponen las teorías cognitivo-evaluativas, es: Percepción-Appraisal-Emoción (Pinedo \& Yáñez, 2017).

Según lo anterior, la perspectiva cognitiva en el estudio de la emoción entiende que la activación de una respuesta emocional está vinculada, básicamente, a los procesos de valoración. Si la valoración del evento es positiva o negativa generará un tipo de patrón psicológico igualmente positivo o negativo que se evidenciará en una expresión característica de una emoción; por ejemplo, en el miedo encontramos sudoración, latidos fuertes y piel de gallina; en este caso el patrón implica la percepción de algo dañino o nocivo en el ambiente. Aunque la afección de entrada es corporal y es provocada por algo externo a nosotros, en el fondo de ella yace algún pensamiento, juicio o creencia relativa a lo que acabamos de percibir, y que nos lo señala como algo temible o digno de atención (Lazarus, 1996).

Una teoría cognitiva de la emoción es, por consiguiente, aquella que incluye algún aspecto del pensamiento, usualmente una creencia o un juicio, como un componente necesario para la reacción emocional. En este texto basta afirmar que en estas posturas el concepto de cognición se entiende de manera amplia y se asocia con la interpretación que damos a cosas o situaciones. El acto de valoración, o el juicio, puede darse de manera elaborada, donde los cálculos racionales y la consciencia explícita participan, o de manera preverbal y prerreflexiva (Lyons, 1993).

En síntesis, este tipo de teorías defienden la intervención de complejos mecanismos cognitivos en la expresión emocional; por tanto, cuestionan y hacen borrosa la tradicional oposición entre razón y emoción que subyace en la filosofía moral moderna. Si la emoción está asociada a cogniciones, creencias y evaluaciones, entonces el juicio moral se puede ver afectado por las valoraciones que hacemos de las situaciones como dignas de compasión, vergüenza, ira o indignación (Roberts, 2003).

\section{Martha Nussbaum en el contexto de las teorías cognitivas contemporáneas de la emoción.}


Martha Nussbaum, considerada una de las pensadoras actuales más notables, se ha convertido en los últimos años en un referente para todos aquellos interesados en comprender las teorías cognitivas de la emoción y sus posibles aplicaciones en diferentes campos de la vida humana. En Paisajes del pensamiento: la inteligencia de las emociones (2008), Nussbaum expone su visión cognitivo-evaluadora de las emociones, la cual es el resultado de una relectura de los planteamientos estoicos sobre las emociones, entendidas como juicios de valor, en combinación con una recuperación del concepto de eudaimonía, propio de la tradición aristotélica. En el pensamiento de Nussbaum (1995, 1996, 1997, 1998, 2005, 2006, 2008), los juicios valorativos juegan un rol esencial para que se produzca la reacción emocional, y es a través de ellos como podemos encontrar una conexión entre emociones y vida moral. Los juicios son propiedades de lo emocional que se sostienen en el cúmulo de creencias que los seres humanos desarrollamos en torno a un sinnúmero de objetos, personas y bienes externos que no están bajo nuestro control, pero que influyen poderosamente sobre nuestra percepción del mundo o en nuestra manera de interpretar el mundo. Esta es la razón por la que Nussbaum acentúa en sus escritos lo que denomina una inteligencia de las emociones: son creencias o juicios que nos hacen implicarnos en el mundo de determinada manera.

Inspirada en Aristóteles y en el estoicismo antiguo ${ }^{3}$, Nussbaum concibe una teoría cognitivo-evaluadora de las emociones que las entiende como un tipo especial de juicios: juicios de valor o evaluaciones que permiten ver el mundo desde el punto de vista de nuestro esquema de objetivos y proyectos; las cosas a las que asignamos valor en el marco de una concepción de lo que para nosotros significa el florecimiento de una vida humana buena (Nussbaum, 2006, 2008, 2014). Si bien su teoría no aborda todos los tipos de emoción, en Paisajes del pensamiento: la inteligencia de las emociones expone una amplia interpretación de la dimensión cognitiva y evaluativa de la compasión, considerada positivamente por la reflexión filosófica como una emoción que ofrece un buen fundamento para las acciones adecuadas desde el punto de vista moral.

En el contexto de las teorías contemporáneas de la emoción, Nussbaum analiza la compasión como un tipo de emoción específicamente humana estructurada por tres elementos cognitivos o filtros que determinan su racionalidad. El primer componente cognitivo o filtro de la compasión es el "juicio de magnitud" o gravedad de la situación; se trata de una valoración o cómputo que permite discriminar el daño o sufrimiento que padece un individuo en determinada circunstancia y que por las mismas condiciones en que acontecen los hechos no es trivial o fácilmente restituible. El segundo es lo que Nussbaum denomina el "juicio de inmerecimiento" o valoración que lleva a considerar el sufrimiento de otro ser humano como no provocado, sin que medie ningún fallo de su parte, sino que es una situación que refleja la radical condición de vulnerabilidad que nos es constitutiva: “Al

\footnotetext{
${ }^{3}$ La influencia estoica y aristotélica en el pensamiento de Nussbaum se encuentra ampliamente desarrollada en su texto La terapia del deseo: teoría y práctica en la ética helenística (2003). Por el objeto y brevedad de este artículo no nos centramos en la lectura que la autora hace de los pensadores antiguos.
} 
sentir compasión por otra persona, la persona compasiva acepta, entonces, cierta representación del mundo, según la cual las cosas valiosas no siempre están a salvo y bajo control, sino que a veces pueden resultar dañadas por la acción de la fortuna" (Nussbaum, 2008, p. 354). El tercero es lo que Nussbaum denomina el juicio eudaimonista o evaluación que el sujeto realiza de la situación en que se encuentra otra persona u objeto intencional, que es asumido como un elemento valioso en su esquema de objetivos y proyectos y un fin en sí mismo, cuyo bien debe ser promovido.

El juicio eudaimonista es una idea que Nussbaum recupera de Aristóteles a partir de una lectura de la Ética Nicomaquea y la Ética Eudemia, la cual le permite asociar las emociones con la noción de vida buena o florecimiento humano, que es fundamental para el desarrollo personal y la vida en sociedad. Este juicio eudaimonista implica, en la mayoría de los casos, otro requisito cognitivo, denominado "juicio de las posibilidades parecidas", que es una forma de imaginación empática que permite identificarse con la persona que sufre, bajo la idea de que compartimos una misma condición humana vulnerable, frágil, sometida a los avatares de la fortuna (Nussbaum, 1995). Para nuestra autora, es en esa frágil condición donde hunde sus raíces la comunidad humana, y la compasión es una inmediata respuesta a ese grito de labilidad que lanzamos por nuestra mortalidad. Este elemento cognitivo permite concebir la compasión como la forma específica que tiene nuestra especie de ligar el bien de los otros a la estructura fundamentalmente eudaimonista, no egoísta, de nuestra imaginación y de nuestras preocupaciones más intensas: "Para que se despierte la compasión se debe considerar el sufrimiento de otra persona como una parte significativa del propio esquema de objetivos y metas. Se deben tomar las penurias de otra persona como algo que afecta el propio florecimiento" (Nussbaum, 2008, p. 258).

Pero si bien es importante atender estos tres filtros gruesos que establecen un marco de condiciones para que se genere la compasión, es justo destacar algunas preguntas y demandas de precisión que se le pueden hacer a la teoría de Nussbaum y que tienen que ver con su comprensión de lo cognitivo y la clasificación de las emociones.

\section{Niveles cognitivos y expresión emocional: problemas y perspectivas que surgen en la teoría de las emociones de Nussbaum.}

Teniendo en cuenta que la creencia es el fundamento de la emoción, Nussbaum postula la posibilidad de cambios en la valoración en la medida en que se modifiquen las creencias que el sujeto tiene sobre determinados objetos intencionales. Esta es una teoría que la filósofa retoma de la tradición estoica en donde se postula una estrecha relación entre emoción y creencia. En Terapia del deseo (2003), Nussbaum desarrolla ampliamente las ideas estoicas de Crisipo y Séneca, según las cuales las pasiones son formas de falso juicio o falsa creencia: 
"Las pasiones no son sacudidas subracionales procedentes de nuestra naturaleza animal, sino modificaciones de la facultad racional; entonces, para ser moderados y poder llegar a curarnos, hemos de enfrentarnos a ellas con una técnica terapéutica que use las artes de la razón" (2003, p. 456). Estas creencias o juicios, que son elementos constitutivos, necesarios, de la emoción, son juicios de valor, es decir, opiniones o suposiciones de lo bueno y lo malo que tienen diversos objetos o bienes externos, que se juzgan de gran importancia para la vida o que se juzgan dignos de ser elegidos como orientadores de la existencia. Por ejemplo, la creencia de que el dinero es el mayor bien y que una vida sin dinero no es digna de ser vivida puede generar en quien no lo tiene, o lo ha perdido, una profunda sensación de tristeza, o la creencia de que la posición social es de gran valor para la felicidad puede generar ira o indignación frente a aquellos que por alguna circunstancia no reconocen en uno a un ser de gran prestancia dentro de la sociedad. En estos ejemplos parece que la noción de creencia, en nuestra autora, hace referencia a ideas que el sujeto puede hacer explícitas, o que son parte de unos conocimientos claramente delimitados $\mathrm{y}$, en muchos casos, compartidos con buena parte de la población.

Inicialmente, y de manera general, bajo la categoría de creencias encontramos diferentes formas de pensamiento: convicciones, dudas, suposiciones, especulaciones, conjeturas, sobre las cuales está fundada una emoción. Pero ¿qué podríamos decir de la creencia que una anoréxica tiene de su propio cuerpo, o de las creencias que una madre sobreprotectora tiene sobre sus hijos y que la llevan a sufrir permanentemente ante hechos que pueden ser interpretados como insignificantes por una gran mayoría?, ¿ese tipo de creencias operan de la misma forma que las expuestas por Nussbaum? o ¿podríamos calificar como una creencia de la misma naturaleza el que una persona se sienta responsable y culpable por comer algún plato exquisito cuando se acuerda de las hambrunas en algún lugar del mundo, o que alguien se sienta causante de un accidente que sufre una persona cercana como consecuencia de no declararle suficientemente su amor? Estos ejemplos nos sugieren la necesidad de ser más precisos con la categoría de creencia o, mejor, nos conducen a una precisión de lo que entendemos por cognitivo y, de manera derivada, a una clarificación de las formas variadas de la expresión de las emociones. Solo de esta manera podremos establecer la particularidad de la compasión y la atribución única de esta emoción a nuestra especie.

En pos de precisar algunos conceptos habremos de entender lo cognitivo de la manera más amplia posible. Cognición hace referencia a las maneras como los organismos seleccionan y valoran la información; con esta concepción podemos atribuir a los animales no humanos formas cognitivas, ya que es fundamental para su supervivencia reconocer ciertos estímulos o eventos del ambiente; una rana puede ser muy sensible a objetos de determinado tamaño que, usualmente en su mundo, corresponden a insectos de los que se alimenta; un animal puede diferenciar un depredador de una presa o de otro animal con el que no corre peligro. Estas formas cognitivas pueden diferenciarse de aquellas propias del 
ser humano, las cuales implican creencias explícitas. Posiblemente, la emoción del miedo ante un predador puede ser similar a aquella que podemos sentir cuando nos cuentan de un peligro inminente, pero, obviamente, la significación cambia. A pesar de que podemos compartir con los animales no humanos emociones como el miedo e incluso algunos estímulos o eventos que las ocasionan, es claro que esas mismas emociones en nosotros pueden generarse por infinidad de eventos que se relacionan con una "creencia" explícita.

Este tipo de diferenciación nos permite establecer una clasificación tanto de procesos cognitivos, como de expresiones emocionales que se encuentran en distintos organismos. Esta vía para diferenciar niveles de complejidad debe rastrearse en las investigaciones de diversos autores contemporáneos que establecen niveles cognitivos superiores o facultades cognitivas complejas que implican procesos de consciencia que son exclusivos del ser humano y que, por tanto, determinan la expresión de ciertas emociones, como la compasión (Ekman, 1984; Eisenberg, 2002; Izard, 1977; Izard et al., 2008; Lewis, 1993).

Si bien podemos reconocer con Nussbaum que hay expresiones emocionales en los animales no humanos y, por lo tanto, mecanismos cognitivos simples (como es el caso de los mamíferos), para evaluar si una situación o estímulo es o no favorable, resulta necesario plantear una serie de criterios diferenciales de lo cognitivo, o niveles de complejidad cognitiva, que permitan establecer la frontera entre las emociones humanas susceptibles de creencias, como la compasión, y otras expresiones emocionales que podemos compartir con diferentes organismos vivos, pero que cualitativamente son distintas. Por ejemplo, insistimos en ello, el miedo lo encontramos en animales no humanos, pero su presencia en el hombre demanda unos mecanismos cognitivos específicos que generan una diferencia en la respuesta emocional (Campos \& Barret, 1984; Frijda, Meanstead \& Bem, 2000; Izard, 1992; Nussbaum, 2005; Piaget, 1969; Roberts, 2003). Este aspecto implicaría desarrollar una diferenciación y clasificación de las emociones según el nivel de complejidad cognitiva o conjunto de capacidades cognitivas que determinan la variabilidad emocional en los diferentes organismos vivos y en el ser humano.

Si bien existen clasificaciones múltiples de las emociones que atienden a su duración, intensidad y expresividad, entre algunos criterios, los planteamientos de Nussbaum nos llevan a buscar respuesta en aquellas propuestas que diferencian las emociones de acuerdo con su complejidad cognitiva (Eich, 2003; Frijda, 2007; Hauser, 2006; Panksepp, 1989, 2007).

Los animales no humanos, como ya lo anotamos, expresan miedo, tristeza, ira u otras emociones que, para algunos autores, también se presentan en los niños pequeños (Casacuberta, 2000; Deight, 2004; Ekman, 1984; Mc Auliffe \& Hauser, 2010). Estas emociones básicas demandan la presencia de estímulos u objetos presentes, se expresan de manera rápida y tienen corta duración; son expresiones emocionales propias de formas 
cognitivas elementales donde el lenguaje y las representaciones no hacen presencia. Mientras las emociones denominadas complejas se presentan en los humanos y demandan procesos de mayor desarrollo cognitivo, como la inclusión de creencias, ideas sobre el funcionamiento del mundo y concepciones acerca de la justicia (Bowlby, 1998; Clark, 2014; Brady, 2014). De esta manera podremos diferenciar la compasión, como emoción moral compleja propia del ser humano, de comportamientos altruistas presentes en niveles inferiores de complejidad cognitiva. Los organismos con funciones cognitivas más elementales podrán tener reacciones emocionales y conductas básicas de preocupación por el semejante, sin embargo, no son una respuesta equivalente a las particularidades de la compasión humana, que exige niveles superiores de elaboración conceptual. Las reacciones automáticas, fisiológicas, propias de especies no humanas, pueden ser interpretadas en algunas teorías sobre la emoción como expresiones emocionales de organismos dotados de niveles elementales de cognición, mientras que las respuestas emocionales que expresan creencias, deseos, prejuicios y pasiones de largo aliento son específicas de la especie humana, debido a su mayor complejidad cognitiva (Hansberg, 1996; Hoffman, 1983, 2002; Izard, 1977; Lewis, 1993, 2000; Ortony \& Turner, 1990; Suddendorf, 2013).

\section{La compasión como emoción moral compleja.}

Para Nussbaum, la compasión es un sentimiento que va asociado directamente con la justicia, y ocupa un lugar central en sus propósitos educativos y en su ideal de construir una nueva cultura política pública. Con la compasión se garantiza que los sujetos se apropien del dolor y el sufrimiento de los otros, lo que hace que sea una emoción moral fundamental o la emoción social básica (Nussbaum, 1996). Por tanto, es una emoción que requiere un conjunto complejo de conceptos relacionados con los valores morales, una conciencia de las distinciones morales, de lo correcto o incorrecto, justo o injusto, y una capacidad adaptativa para interpretar correctamente el estado mental de alguien que puede influir en nuestro bienestar (Haidt, 2003; Knobe, 2005; Lewis, 2000; Neblett, 1981).

Las ideas sobre las que se nutre Nussbaum (2008) para desarrollar su idea de compasión nos remite a las reflexiones sobre benevolencia elaboradas por Hume, en su Investigación sobre los principios de la moral, y por Smith, en su Teoría de los sentimientos morales, al igual que a diversos debates contemporáneos sobre el altruismo, los comportamientos de cooperación y las conductas prosociales. Sin desconocer los sentimientos de empatía y las conductas altruistas en otras especies, la noción de compasión planteada por Nussbaum nos lleva a indagar por lo específico de esta emoción en el ser humano y por los mecanismos que hacen posible que esta emoción sea sensible a las formas cognitivas más elaboradas, mecanismos que justificarían cómo las personas pueden corregir sus emociones a partir de la 
educación, los nuevos aprendizajes y la incorporación de nuevas concepciones razonables sobre la vida buena.

En líneas generales, una perspectiva que abogue por una integración particular de la emoción y la cognición, tal y como propone Nussbaum, debe tener en cuenta que para que una emoción sea moral debe implicar la consideración de estándares morales y normativos, es decir, involucrar creencias sobre el quebrantamiento de normas y, a la vez, relacionarse con los estereotipos inherentes a los códigos y creencias individuales (Mercadillo, Díaz \& Barrios, 2007). El que una emoción surja en relación con principios o estándares morales específicos significa que involucra elementos cognitivos superiores para su generación, esto es, una serie de conceptos, creencias y deseos relacionados con la moralidad, un conocimiento del otro y un reconocimiento de los diversos matices del sistema de relaciones interpersonales, con las exigencias y las expectativas que estas relaciones suponen (Hansberg, 1996). Las emociones morales, como la compasión, pueden catalogarse como complejas ${ }^{4}$, en tanto requieren este tipo de habilidades cognitivas superiores, y se pueden denominar emociones autoconscientes $^{5}$, en tanto parecen surgir como resultado de algún tipo de evaluación del propio self ${ }^{6}$. El resultado de esta evaluación es el que usualmente indica la adecuación de la conducta con los estándares de la moralidad y desencadena la experiencia de emociones como la culpa, la vergüenza, la indignación y la compasión, las cuales han sido, por excelencia, vistas como morales (Eisenberg, 2000).

Estas ideas relacionadas con la moralidad, con el daño a otros, con la posibilidad de sufrimiento y con la noción de justicia, que estructuran el contenido de las emociones morales, se pueden comprender con más detalle en el análisis que Nussbaum realiza de una emoción negativa que obstaculiza el florecimiento humano de individuos y comunidades y frena las posibilidades de desarrollo de lo que, en general, podemos considerar como una buena vida. Se trata de lo que la filósofa denomina el asco y la subordinación de grupos como problema moral.

\footnotetext{
${ }^{4}$ La distinción entre emociones primarias (p. ej. ira, tristeza y miedo) y emociones complejas no se abordará aquí más allá de lo ya dicho, dado que se sale de nuestro objetivo expositivo. Es suficiente con señalar que la diferencia entre estas dos categorías se centra en el nivel de los procesos cognitivos que involucren, y que las emociones morales requieren, obviamente, un conocimiento explícito de reglas y principios éticos. Sin embargo, también resulta importante señalar que las emociones primarias, aunque no estén fundamentadas en esquemas morales, también pueden estar relacionadas con la moralidad en una variedad de formas. Estos aspectos no van a ser abordados en este texto, pero el lector puede ampliar la información en Hoffman (2002) e Izard et. al. (2008).

${ }^{5}$ La etiqueta de "autoconsciente" se deriva del hecho de que la experiencia de emociones como la culpa y la vergüienza involucra un proceso de pensamiento reflexivo sobre el self (Eisenberg, 2000).

${ }^{6}$ Por lo que se cree que no se encuentran en animales no humanos, sin capacidad de autorreflexión, ni en niños pequeños, quienes aún no han adquirido la capacidad de pensar conscientemente acerca de ellos mismos (Lewis, 1993; Leary, 2004).
} 
Si la compasión es la emoción que nos vincula en un tipo de amor por los demás, la repugnancia divide y genera asco frente a lo diferente. El asco "hace peligrar los proyectos personales o nacionales que implican algún tipo de sacrificio altruista por el bien común, ya que divide la vida pública en grupos ordenados jerárquicamente que no deben coincidir entre sí” (Nussbaum, 2014, p. 255). Este aspecto del asco lo analiza Nussbaum con mayor detenimiento en El ocultamiento de lo humano; nos dice la filósofa que la repugnancia surge de la creencia en la propiedad contaminante de ciertos objetos que nos recuerdan nuestra naturaleza animal y nuestra mortalidad: fluidos, secreciones, viscosidades, desechos. En esta emoción subyace, por tanto, la idea según la cual "si absorbemos o entramos en contacto con cosas en descomposición, seremos mortales y estaremos en estado de descomposición" (2006, p. 110). No solo los objetos nos dan asco, cuya manifestación más visible es el vómito, sino también la "historia social de los objetos", es decir, aquellas cosas con las cuales creemos que ese objeto estuvo en contacto. Desarrollamos entonces la creencia de que si entramos en contacto con ese objeto o, peor aún, si lo ingerimos, su naturaleza degradada nos contaminará hasta lo más profundo, es decir, hasta nuestro yo o estructura mental.

Si recordamos, en la perspectiva cognitiva de Nussbaum, las creencias configuran la valoración que hacemos de personas, objetos y situaciones como positivas o amenazantes, de tal forma que los juicios son los moduladores de la respuesta emocional. En el caso que analizamos sabemos, desde una perspectiva evolutiva, que el asco tiene una función positiva: nos alerta sobre alimentos y objetos peligrosos para nuestra salud y bienestar; pero desde un punto de vista político y social se convierte en la fuente principal de humillación hacia otros. De esta forma surge lo que Nussbaum denomina la repugnancia extendida y la subordinación de los grupos: extendemos hacia otros seres humanos la idea de que son elementos contaminantes que no deben estar cerca de nosotros. Lo primero que hace la repugnancia es generar una exacerbación de valores equivocados; por ejemplo, frente al patriotismo, genera lo contrario, que es la aversión y la división de clases, grupos y etnias. La repugnancia genera creencias que ciertas personas (homosexuales, afrodescendientes, pobres o discapacitados, entre otros) traen consigo la contaminación para nuestra "alma transparente" y, por tanto, la solución es la discriminación de esos grupos. La repugnancia, en perspectiva de Nussbaum, es antiliberal, antidemocrática y antipluralista; es la emoción que antecede al odio y la humillación, y en diversas circunstancias ha sido causa de grandes males en la humanidad, porque excluye y alimenta la injusticia social (Nussbaum, 2014).

Junto a la repugnancia suele caminar la vergüenza, esa emoción que invita a estigmatizar los rostros de miles de seres humanos en virtud de su condición marginal, desposeída y frágil. Nussbaum, en El ocultamiento de lo humano (2006), habla de una historia natural de la vergüenza y la humillación como narrativa de las causas más profundas por las que las sociedades humanas, una y otra vez, buscan marcar los rostros de algunos de sus miembros, lo que conduce a vivir con una "identidad manchada". La vergüenza busca, fundamentalmente, hacer sentir a ciertos miembros de la comunidad como indignos de 
pertenecer a una colectividad, grupo o nación, porque las personas en cuestión "son rastreras, no están a la par de otros en términos de dignidad humana" (Nussbaum, 2006, p. 241).

No son muy distinguibles las fronteras entre avergonzar y humillar; por lo general, se avergüenza para provocar humillación o se humilla para provocar vergüenza. Lo cierto es que se estigmatiza a las personas como no humanas, no dignas, no capaces, no puras, no "normales", no limpias; como seres contaminantes que traen consigo algo degradante que es mejor mantener a distancia. Ejemplos hay muchos, pero siguiendo las ideas de Nussbaum, las minorías LGBTI, los afroamericanos, los discapacitados, los individuos que sufren alguna deformidad, los pobres, son casos de individuos vulnerables que fácilmente pueden ser presa de la estigmatización social que conduce a la vergüenza. Esta dinámica de denigración se vuelve más aguda cuando en el derecho y en las legislaciones se incluye el factor emocional de la repugnancia como criterio para tratar a ciertos individuos, normalmente delincuentes o transgresores de la ley, como seres que ya no tienen redención y que difícilmente merecen ser contados como pertenecientes a la especie humana (Pinedo \& Yáñez, 2017).

En este punto de la argumentación destacamos el papel que tiene la vía emocional como una herramienta para detectar daños morales. La presencia del asco y la vergüenza nos deben conducir a un rechazo y desaprobación, por ser actitudes moralmente injustificables. Tal como concibe Nussbaum el juicio eudaimonista, las emociones incluyen valoraciones acerca de cómo se ve una cosa o una persona y cómo se cree que su presencia puede afectar nuestros objetivos, planes y a nosotros mismos; esto ya es relevante en un sentido moral, pues relaciona directamente las emociones con la percepción de posibles daños por valoraciones negativas, de repulsa, de aversión del sujeto hacia individuos y grupos, actitudes que nutren las xenofobias y muchas otras formas de discriminación (Nussbaum et al., 2007).

Como vamos dilucidando en esta reflexión, la teoría de las emociones de Martha Nussbaum es muy amplia y rica en matices, lo cual es supremamente valioso para el actual debate sobre la relación entre emociones y vida moral. Sabemos que la compasión es una emoción con alto contenido ético, idea que ya encontramos en los moralistas británicos y también en Schopenhauer, que afirma la existencia humana como un padecer, como un sufrimiento ineludible que nos impulsa a compadecernos de los demás seres humanos, vistos como compañeros de camino amenazados por esa irrefrenable voluntad que subyace en todo lo que existe. No obstante, la tesis de Nussbaum de que hay una inteligencia de las emociones nos orienta a una comprensión de la compasión bastante original y significativa para el mundo actual. De hecho, Nussbaum se considera a sí misma una autora que concibe el quehacer filosófico en la línea propuesta por las escuelas helenísticas antiguas: la filosofía debe ser una medicina para el ser humano y sus problemas, es una terapia frente a un mundo hostil, complejo y muchas veces incomprensible. La compasión en esta perspectiva conduciría a lo que Nussbaum denomina, en Emociones políticas (2014), el surgimiento y la puesta en marcha de una "ciudadanía compasiva" en donde la suerte de los demás, particularmente de 
los más desvalidos, cuenta, y cuenta no solo como un sentimiento individual, sino como una emoción que exige a los organismos de gobierno una preocupación concreta, desde las leyes, desde las políticas públicas, por aquellos excluidos, estigmatizados, empobrecidos y humillados por sistemas injustos y ampliamente inequitativos.

\section{CONCLUSIONES.}

Después de largos años en los que la dicotomía razón-emoción se había apropiado de los tratados filosóficos sobre la naturaleza de las pasiones, hoy en día teorías cognitivoevaluativas, como la de la filósofa norteamericana Martha Nussbaum, reorientan la comprensión de las emociones hacia otros derroteros de reflexión. Nussbaum nos invita a concebir las emociones como reacciones dotadas de un componente cognitivo-racional que encarna maneras de interpretar el mundo: las emociones van ligadas a creencias o juicios que nos hacen ver el mundo de determinada forma; estas creencias comprenden de manera destacada nuestras ideas valorativas acerca de lo que es bueno y malo, meritorio o sin mérito, favorable o perjudicial. Esta valoración nos lleva a considerar como de gran relevancia muchos acontecimientos en los cuales nos encontramos inmersos día tras día, estableciendo un complejo entramado de conexiones entre el agente y los objetos mediante consideraciones circunstanciales y de perspectiva que nos hacen finalmente reaccionar de determinada manera. Si los eventos son interpretados como perjudiciales, nos generan temor; si los consideramos una afrenta para nosotros o para alguien amado, nos producen ira o indignación; también sentimos envidia si queremos o apreciamos un objeto y no lo tenemos, pero otro fácilmente lo tiene; lo que tememos para nosotros mismos nos produce compasión cuando le sucede a otro.

Esta visión cognitivo-valorativa de las emociones fundamentadas en creencias posibilita establecer una conexión entre emociones y vida moral. Las emociones son juicios que pueden estar asociados a nuestras concepciones morales, de ahí que en filosofía y psicología se hable de emociones morales o emociones con contenido moral. Compasión, culpa e indignación son emociones características de nuestro mayor desarrollo ontogenético y, tal como nos indica el estado actual de la investigación, son específicamente humanas, no pertenecen al repertorio emocional de otras especies. Queda por comprender más a fondo qué tipo de facultades cognitivas entran en juego para que se den este tipo de emociones morales, cuestión que hace parte del estado del arte que dejamos abierto para un ulterior desarrollo.

En esta misma línea de reflexión, la teoría cognitiva de Nussbaum nos lleva a comprender las emociones como informadoras de sufrimiento, daño moral y dignidad humana menoscabada, asunto que resulta de gran relevancia para la comprensión de nuestros juicios morales. Si no tuviéramos un repertorio emocional, difícilmente valoraríamos las acciones 
de otras personas y las propias desde la óptica del daño o atropellamiento a la dignidad, es decir, bajo la creencia de que hay acciones inaceptables, despreciables o crueles que no deberían ocurrir porque degradan al ser humano. Nuestro sistema emocional es, pues, un dispositivo de alerta contra abusos, violencia o sufrimiento que nos permite no solo interpretar diversas situaciones externas a nosotros, sino también ponernos en el lugar de cualquier persona afectada y ver todo lo que puede suceder cuando no se reacciona adecuadamente frente a la injusticia y la opresión.

\section{REFERENCIAS}

Arnold, M. (1960). Emotion and Personality. New York: Columbia University Press.

Ben Ze'ev, A. (2000). The Subtlety of Emotions. Cambridge: MIT Press.

Bowlby, J. (1998). El apego y la pérdida. Barcelona: Paidós.

Brady, M. (2014). Emotional Insight. The Epistemic Rol of Emotional Experience. Oxford: Oxford University Press.

Cabezas, M. (2014). Ética y emoción. El papel de las emociones en la justificación de nuestros juicios morales. Madrid: Plaza y Valdés.

Calhoun, C. \& Solomon, R. (1989). ¿Qué es una emoción? Lecturas clásicas de psicología filosófica. México: Fondo de Cultura Económica.

Campos, J. J. \& Barret, K. C. (1984). Toward a new understanding of emotions and their development. En Izard, C. E., Kagan, J. \& Zajonc, R. B. (eds.), Emotions, Cognition \& Behavior (pp. 229-248). Cambridge: Cambridge University Press.

Casacuberta, D. (2000). ¿Qué es una emoción? Barcelona: Crítica.

Clark, M. S. (2014). Prosocial Behavior. En Malmoli, S. (ed.). McGraw Hill Yearbook of Science and Technology. New York: McGraw Hill.

Cornelius, R. (1996). The Science of Emotions. New Jersey: Prentice Hall.

De Waal, F. (2009). Primates and Philosophers: How Morality Evolved. Princeton: Princeton University Press.

Deight, J. (2004). Primitive Emotions. En Solomon, R. (ed.). Thinking About Feeeling. Oxford: Oxford University Press.

Dixon, T. (2003). From Passions to Emotions. The Creation of a Secular Psychological Category. New York: Cambridge University Press. 
La dimensión cognitiva de las emociones en la vida moral: los aportes de Martha Nussbaum al estado de la discusión. Iván A. Pinedo-Cantillo y Jaime Yañes-Canal. Pág.: 105 - 127

Dixon, T. (2010). Thomas Brown. Selected Philosophical Writings. Charlottesville: Academic Philosophy Documentation Center.

Eich, E. (2003). Cognición y emoción. Bilbao: Desclée de Brouwer.

Eisenberg, N. (2000). Emotion, regulation, and moral development. Annual Review of Psychology, 51, 665-697.

Eisenberg, N. (2002). Empathy-Related Emotional Responses, Altruism, and their Socialization. En Davidson, R.J. \& Harrington, A. (eds.). Visions of Compassion (pp. 131-164). Oxford, UK: Oxford University Press.

Ekman, P. (1984). Expression and the nature of emotion. En Scherer, K \& Ekman, P. (eds.). Approaches to emotion (pp. 319-344). Hillsdale, N. J.: Lawrence Erlbaum.

Ekman, P., y Davidson, R. (1994). The Nature of Emotions. New York: Oxford University Press.

Frijda, N. (1987). Emotions. Cambridge: Cambridge University Press.

Frijda, N., Meanstead, A.S. \& Bem, J. (eds.). (2000). Emotions and beliefs. How feelings influence thoughts. Cambridge: Cambridge University Press.

Haidt, J. (2003). The Moral Emotions. En Davidson, R.J., Scherer, K. \& Goldsmith, H. (eds.). Handbook of Affective Sciences (pp. 852-870). Oxford: Oxford University Press.

Hansberg, O. (1996). De las emociones morales. Revista de Filosofía. $3^{\circ}$ época, vol. IX, núm. 16, pp. 151-170. Madrid: Servicio de Publicaciones, Universidad Complutense.

Hauser, M. (2006). Moral Minds. How nature designed our universal sense of right and wrong. New York: Harper Collins.

Hoffman, M. (1983). Affective and Cognitive Processes in Moral Internalization: an Information Processing Approach. En Higginss, E. (ed.). Social Cognition and Social Development (pp. 236-274). Cambridge: Cambridge University Press.

Hoffman, M. (2002). Desarrollo moral y empatía. Barcelona: Idea Book S.A.

Valenzuela, A. (2016). El gobierno de las emociones. Cuestiones de Filosofía, (18) 1. P. 215217.

Hume, D. (1985). Tratado de la naturaleza humana. (V. Viqueira, Trad.). México: Porrúa.

Hume, D. (2004). Disertación sobre las pasiones y otros ensayos morales. Barcelona: Anthropos.

Izard, C. E. (1977). Human Emotions. New York: Plenum Press. 
Izard, C.E. (1992). Basic Emotions, Relations among Emotions, and Emotion-Cognition Relations. Psychological Review, 99, 561-565.

Izard, Stark, Trentacosta \& Schultz (2008). Beyond Emotion Regulation: Emotion Utilization and Adaptive Functioning. Child Development perspectives, 2 (3), 156163.

James, W. (1884). ¿Qué es una emoción? Estudios de psicología, 21, $57-73$.

Kant, E. (2013). Fundamentación de la metafísica de las costumbres. México: Porrúa.

Knobe, J. (2005). Theory of Mind and Moral Cognition: Exploring the Connections. Trends in Cognitive Sciences, 9(8), 357-359.

Lazarus, R. (1991). Emotion and adaptation. New York: Oxford University Press.

Lazarus, (1996). Passion and Reason. Making sense of our emotions. Oxford: Oxford University Press.

Leary, M. (2004). Digging Deeper. The Fundamental Nature of "Self-Conscious" Emotions. Psychological Inquiry, 15(2), 129-131.

LeDoux, J. (1999). El cerebro emocional. Barcelona: Planeta.

Lewis, M. (1993). The emergence of human emotions. En Lewis, M., \& Haviland-Jones, J. (eds.), Handbook of emotions (pp. 265-280). Nueva York: The Guilford Press.

Lewis, M. (2000). Self-conscious emotions: embarrassment, pride, shame and guilt. En Lewis, M., \& Haviland-Jones, J. (eds.), Handbook of emotions, 2a Ed. (pp. 623-636). New York: The Guilford Press.

Lyons, W. (1993). Emoción. Barcelona: Anthropos.

Mc Auliffe, K. \& Hauser, M. (2010). Morality and Evolution. Encyclopedia of Animal Behavior (pp. 483-488).

Mercadillo, Díaz \& Barrios. (2007). Neurobiología de las emociones morales. Salud Mental, 30(3), 1-11.

Neblett, W. (1981). The Role of Feelings in Morals. Washington: University Press of America.

Nussbaum, M. (1995). La fragilidad del bien. Fortuna y ética en la tragedia y la filosofía griegas. Madrid: Visor. 
La dimensión cognitiva de las emociones en la vida moral: los aportes de Martha Nussbaum al estado de la discusión. Iván A. Pinedo-Cantillo y Jaime Yañes-Canal. Pág.: 105 - 127

Nussbaum, M. (1996). Compassion: the basic social emotion. Social Philosophy and Policy, 13(1), 27-58.

Nussbaum, M. (1997). Emotions as Judgment of Value and Importance. En Bilimoria, P. \& Mohanty, J.N. (eds.). Relativism, Suffering, and Beyond: Essays in Memory of Bimal K. Matilal (pp. 231-251). Delhi: Oxford University Press.

Nussbaum, M. (1998). Emotions as Judgment of Value: a Philosophical Dialogue. Comparative Criticism, fall, 1-30.

Nussbaum, M. (2003). La terapia del deseo: teoría y práctica en la ética helenística. Barcelona: Paidós.

Nussbaum, M, (2005). Emotions and the Origins of Morality. Advances in Psychology, 137, 61-117.

Nussbaum, M. (2006). El ocultamiento de lo humano. Repugnancia, vergüenza y ley. Buenos Aires: Katz.

Nussbaum, M., Chan, J., Lau, J. \& Jiwei, C. (2007). The Ethics and Politics of Compassion and Capabilities. Hong Kong: Faculty of Law, University of Hong Kong.

Nussbaum, M. (2008). Paisajes del pensamiento: la inteligencia de las emociones. Barcelona: Paidós.

Nussbaum, M. (2014). Emociones políticas. ¿Por qué el amor es importante para la justicia? Barcelona: Paidós.

Ortony, A. \& Turner, T. J. (1990). What's basic about basic emotions? Psychological Review, 97, 315-331.

Panksepp, J. (1989). The neurobiology of emotions: Of animal brains and human feelings. En Wagner, H. \& Manstead, T. (eds.). Handbook of social psychophysiology (pp. 526). Chichester: Wiley.

Panksepp, J. (2007). Criteria for basic emotions: Is disgust a primary “emotion'? Cognition and Emotion, 21(8), 1819-1828.

Piaget, J. (1969). Biología y conocimiento. Madrid: Siglo XXI.

Pinedo, I. \& Yáñez, J. (2017). Las emociones y la vida moral: una lectura desde la teoría cognitivo-evaluadora de Martha Nussbaum. Veritas, 36, 47-72.

Plamper, J. (2015). The History of Emotions. An Introduction. Oxford: Oxford University Press. 
Plutchik, R. (1980). Emotion. Theory, Research, and Experience. Vol. 1, Theories of Emotion. New York: Academic Press.

Roberts, R. (2003). Emotions. And Essay in Aid of Moral Psychology. Cambridge: Cambridge University Press.

Roberts, R. (2013). Emotions in the Moral Life. Cambridge: Cambridge University Press.

Seoane, J. (2004). Del sentido moral a la moral sentimental. El origen sentimental de la identidad y ciudadanía democrática. Madrid: Siglo XXI.

Solomon, R. (2003). Not passion's Slave: Emotions and Choice. New York: Oxford University Press.

Suddendorf, T. (2013). The gap: The science of what separates us from other animals. New York: Basic Books. 